\title{
Diagnostic Accuracy of Hepatic Vein Arrival Time Performed with Contrast-Enhanced Ultrasonography for Cirrhosis: A Systematic Review and Meta-Analysis
}

\author{
Gaeun $\mathrm{Kim}^{1}$, Kwang Yong Shim ${ }^{2}$, and Soon Koo Baik ${ }^{2}$ \\ ${ }^{1}$ Department of Nursing, Keimyung University College of Nursing, Daegu, and ${ }^{2}$ Department of Internal Medicine, Wonju Severance Christian \\ Hospital, Yonsei University Wonju College of Medicine, Wonju, Korea
}

See editorial on page 9.

Background/Aims: We identified reports in the literature regarding the diagnostic accuracy of hepatic vein arrival time (HVAT) measured by contrast-enhanced ultrasonography (CEUS) to assess hepatic fibrosis in cirrhosis. Methods: The Ovid MEDLINE, Embase, and Cochrane databases were searched for all studies published up to 23 July 2015 that evaluated liver status using CEUS and liver biopsy (LB). The QUADAS-II (quality assessment of diagnostic accuracy studies-II) was applied to assess the internal validity of the diagnostic studies. Selected studies were subjected to a meta-analysis with MetaDisc 1.4 and RevMan 5.3. Results: A total of 12 studies including 844 patients with chronic liver disease met our inclusion criteria. The overall summary sensitivity, specificity, positive likelihood ratio, and negative likelihood ratio of the HVAT measured by CEUS for the detection of cirrhosis compared to LB were 0.83 (95\% confidence interval [Cl], 0.77 to 0.89$), 0.75(95 \% \mathrm{Cl}, 0.69$ to 0.79$), 3.45$ (95\% Cl, 1.60 to 7.43$)$, and $0.28(95 \% \mathrm{Cl}, 0.10$ to 0.74$)$, respectively. The summary diagnostic odds ratio (random effects model) was 15.23 (95\% Cl, 3.07 to 75.47$)$, the summary receiver operator characteristics area under the curve was 0.74 (standard error $[S E]=0.14$ ), and the index $Q$ was 0.69 ( $\mathrm{SE}=0.11$. Conclusions: Based on a systematic review, the measurement of HVAT by CEUS exhibited an increased accuracy and correlation for the detection of cirrhosis. (Gut Liver 2017;11:93-101)
Key Words: Contrast-enhanced ultrasonography; Hepatic fibrosis; Meta-analysis; Systematic review

\section{INTRODUCTION}

Cirrhosis is the end stage of chronic liver disease and is characterized by regenerative nodules with extensive surrounding fibrosis. The distortion of architecture caused by the structural changes leads to portal hypertension and associated serious complications, such as variceal bleeding and ascites..$^{1-7}$

Estimating the degree of hepatic fibrosis is important for diagnosis and therapeutic management of these patients; at present, liver biopsy is the gold standard method. However, only a small fraction of the liver parenchyma is sampled in this process, and the biopsy procedure is associated with a small but definite risk of morbidity and mortality. Hence, the development of a noninvasive technique for assessing liver disease is much needed. ${ }^{2,3,6,8}$

Ultrasound, which is widely used as the initial imaging test, is insufficient as an accurate means of assessing disease severity. A number of Doppler ultrasound-based methods have been developed, mainly to measure the relative flow or velocity in the portal vein and the hepatic artery or vein. However, there is controversy with regard to the reproducibility of these studies, and many do not permit investigation or grading of precirrhotic liver disease. ${ }^{2,3}$ Contrast-enhanced ultrasonography (CEUS) is currently considered to be an imaging modality offering new perspectives in the diagnosis of liver disease. ${ }^{9,10}$ The recently developed technique of CEUS has markedly expanded the possibilities for sonographic hemodynamic studies. The technique

\footnotetext{
Correspondence to: Soon Koo Baik ${ }^{\mathrm{a}}$ and Kwang Yong Shim ${ }^{\mathrm{b}}$

a Department of Internal Medicine, Yonsei University Wonju College of Medicine, 20 Ilsan-ro, Wonju 26426, Korea

Tel: +82-33-741-1223, Fax: +82-33-745-6782, E-mail: baiksk@yonsei.ac.kr

${ }^{b}$ Department of Internal Medicine, Yonsei University Wonju College of Medicine, 20 Ilsan-ro, Wonju 26426, Korea

Tel: +82-33-741-1202, Fax: +82-33-745-6782, E-mail: kyshim@yonsei.ac.kr

Received on January 13, 2016. Revised on March 7, 2016. Accepted on March 14, 2016. Published online August 19 , 2016

pISSN 1976-2283 eISSN 2005-1212 https://doi.org/10.5009/gnl16031

@ This is an Open Access article distributed under the terms of the Creative Commons Attribution Non-Commercial License (http://creativecommons.org/licenses/by-nc/4.0) which permits unrestricted non-commercial use, distribution, and reproduction in any medium, provided the original work is properly cited.
} 
involves the intravenous administration of minute, gas-filled microbubbles that strongly enhance intravascular flow signals. ${ }^{10-12}$ Measurement of hepatic vein arrival (transit) times (HVAT or HVTT) using a microbubble agent, such as Levovist (Schering AG, Berlin, Germany), SonoVue (Bracco SpA, Milan, Italy), or Sonazoid (GE Healthcare, Oslo, Norway) has been suggested as an ultrasound-based technique to assess the degree of hepatic fibrosis based on the hypothesis that the arrival time of the injected microbubble would be shortened in cirrhosis due to hemodynamic abnormalities, such as arteriovenous shunting or capillarization of the sinusoid. , $3,11,12^{2}$

Several groups have previously noted that contrast enhancement of the hepatic vein begins earlier in cirrhotic patients than it does in noncirrhotic subjects, thereby indicating that CEUS could be used to stage and grade liver disease and distinguish between mild and moderate-to-severe forms of hepatitis and cirrhosis. ${ }^{9-13}$ However, the reported accuracy and usefulness have been inconsistent across studies. Systematic review (SR) and meta-analysis (MA) have been shown to enable objective analyses of existing evidence. ${ }^{14-18}$

We conducted a SR and MA to evaluate the diagnostic accuracy of HVAT for detecting cirrhosis and evaluated the correlation between HVAT and severity of chronic liver disease.

\section{MATERIALS AND METHODS}

\section{Study strategy}

A systematic search was undertaken among studies published before July 2015 by two independent reviewers, with disagreements resolved by consensus. Databases were searched with a combination of Medical Subject Headings (MeSH) terms and text words for the population and the index tests, and Boolean operators were used. We searched three main databases (i.e., Ovid MEDLINE, Embase, and the Cochrane library) with no restriction on language or publication status. This study was conducted according to the Cochrane Handbook for Systematic Reviews of interventions ${ }^{19}$ and the statement by the Preferred Reporting Items for Systematic Reviews and Meta-Analyses Group. $^{20}$

\section{Inclusion and exclusion criteria}

Studies were included if they met the following criteria: (1) types of participants: chronic liver disease; (2) type of index test: HVAT performed with CEUS; no restrictions regarding contrast; (3) type of reference standard test: liver biopsy; (4) types of outcome variables: data available on diagnostic accuracy; and (5) type of studies: randomized controlled trials or controlled studies. Animal experiments, chemistry, or cell-line studies and editorials, commentaries, review articles and case reports were excluded.

Studies were selected based on the inclusion/exclusion criteria by reviewing the title and abstract of each study after duplicate articles were removed from the primary search. The remaining studies were confirmed with the original and were validated by applying the inclusion and exclusion criteria. Two independent authors reviewed the results of the search using the inclusion criteria. Disagreements between authors were resolved by discussion.

\section{Methodological quality assessment}

The methodological quality of the selected studies was examined using the QUADAS-II (quality assessment of diagnostic accuracy studies-II), ${ }^{21}$ which uses 11 items to evaluate study quality. Each item is scored "-” if reported, “+” if not reported, or "? (unclear)" if there is no adequate information in the article. Two of the authors independently assessed bias; any disagreement or misunderstandings were resolved by discussion until a consensus was reached.

Publication bias was assessed using Begg's funnel plots and test ( $p>0.05$ was considered to indicate that there was no potential publication bias). ${ }^{22}$

\section{Data extraction and statistical methods}

Data were extracted by two reviewers. All differences in opinion with regard to the data were resolved by discussion until a consensus was reached. Related variables were assessed as both dichotomous data and as continuous data.

Based on the values of true positive (TP), true negative (TN), false positive (FP), and false negative (FN), we calculated the pooled sensitivity and specificity, positive and negative likelihood ratios (LR), and diagnostic odds ratio (DOR). The calculated statistics were used to examine the accuracy of HVAT performed with CEUS for the diagnosis of cirrhosis. All statistics were reported as point values with 95\% confidence interval (CI). Sensitivity was defined as the TP rate and calculated as TP/ $(\mathrm{TP}+\mathrm{FN})$. Specificity was defined and calculated as TN/(FP+TN). LR indicates how much the given test increased or decreased the probability of having the disease. The DOR is a single overall indicator of diagnostic performance and expresses the extent to which the odds of having the disease is greater for people with a positive test result than for people with a negative test result. The DOR was calculated as $(\mathrm{TP} \times \mathrm{TN}) /(\mathrm{FP} \times \mathrm{FN})$. In addition, summary receiver operator characteristics (SROC) curves were constructed to examine the interaction between sensitivity and specificity. We used the area under the curve (AUC) to measure the overall performance of the diagnostic test. ${ }^{23}$ Statistical analyses were performed using Meta-DiSc statistical software version 1.4 (Unit of Clinical Biostatistics team of the Ramón y Cajal Hospital, Madrid, Spain) and RevMan 5.3 (The Cochrane Collaboration, Copenhagen, Denmark ${ }^{19}$ to analyze the accuracy of CEUS.

Evaluation of the numerical values of AUC were as follows: $\mathrm{AUC}=0.5$ was a noninformative test: $0.5<\mathrm{AUC} \leq 0.7$ was a less accurate test: $0.7<$ AUC $<1$ was a very accurate test: and $\mathrm{AUC}=1$ 


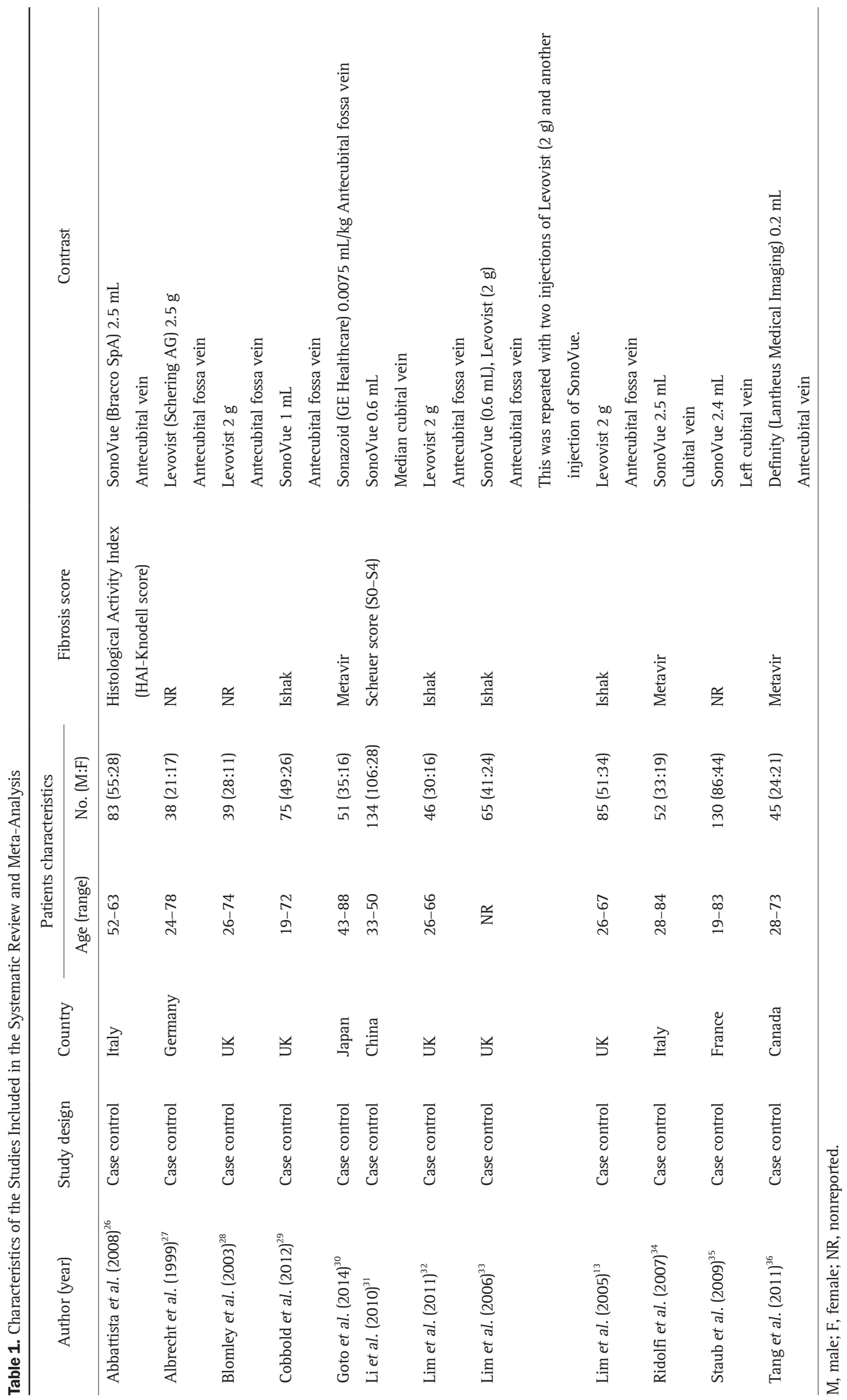


was a perfect test. ${ }^{24}$ Index Q, which represents the correspondence of the specificity and sensitivity of the receiver operating characteristic curve, was evaluated with "1" as the standard at $100 \%$ accuracy. ${ }^{25}$

The between-study heterogeneity was evaluated by computing Higgins $\mathrm{I}^{2}$ and chi-square tests for heterogeneity using the generic inverse variance method of MA. A random effects model was used for statistical pooling of the data in the case of heterogeneity between studies $(\mathrm{p}<0.1)$; a fixed effects model for statistical pooling of the data was used if there was no heterogeneity between the studies.

\section{RESULTS}

\section{General characteristics of the selected studies}

Our initial literature search yielded 208 references. After excluding 26 duplicate studies, a total of 182 studies remained. The titles and abstracts of these studies were reviewed; 170 were excluded based on the selection criteria, and 12 were ultimately identified as relevant to our review. Therefore, we analyzed these 12 studies $^{13,26-36}$ and the 844 included patients, as shown in Table 1 and Fig. 1.

Five of the 12 studies $^{13,28,29,32,33}$ were conducted in the United Kingdom, and the remainder of the studies was conducted in the Italy, Germany, France, Canada, Japan, or China. The in- cluded studies were published between 1999 and 2014. The subjects of study were mainly patients with hepatitis $C$ virus or hepatitis B virus. The number of study subjects in each study ranged from 38 to $134,{ }^{27,31}$ but most of the studies had fewer than 100 subjects. They were case control studies that compared a group of patients that had confirmed cirrhosis through liver biopsy, based on the reference standard, to a control group. All liver biopsies were performed by skilled doctors, and the basic principles of biopsy were faithfully assured. Seven studies used a Siemens Sequoia ultrasound system (Siemens Healthcare, Erlangen, Germany). ${ }^{13,27,28,31-33,35}$ GE Logiq system (General Electric Healthcare, Milwaukee, WI, USA) was used in two of the studies, ${ }^{26,34}$ while a Toshiba system (Toshiba Corporation, Tokyo, Japan) was used in two other studies. ${ }^{29,30}$ With regard to contrast, there were five studies ${ }^{13,27,28,32,33}$ that used Levovist, the first-generation contrast agent. Six studies ${ }^{26,29,31,33-35}$ used the second-generation SonoVue. Sonazoid and Definity (Lantheus Medical Imaging, North Billerica, MA, USA) were each used in one study. ${ }^{30,36}$ As for the amount of contrast used, most studies used 2 to $2.5 \mathrm{~g}$. The HVAT was measured using the Doppler test in nine of 12 studies, ${ }^{13,26,28,30-34,36}$ and the remaining three studies were assessed by visual inspection. ${ }^{27,29,35}$ Although the standard for cirrhosis varied from study to study, Metavir ${ }^{30,34,36}$ or Ishak ${ }^{13,29,32,33}$ was generally used and labeled stages 5 to 6 or F4, according to the fibrosis score, as cirrhosis. Other methods,
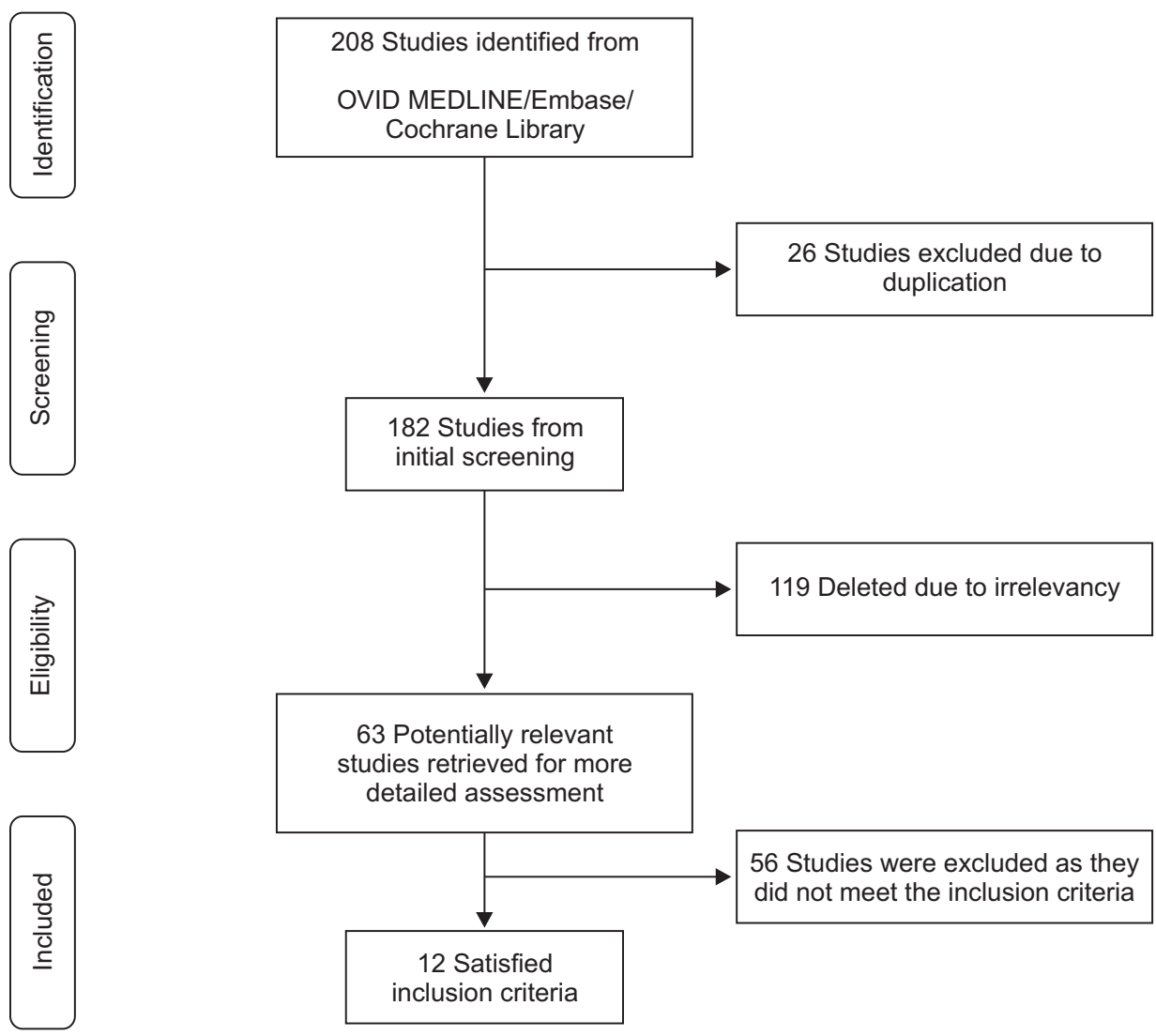

Fig. 1. Flow diagram of study selection for the systematic review. 
including the Histological Activity Index (HAI-Knodell score) ${ }^{26}$ and Scheuer score (S0 to S4), ${ }^{31}$ were also used in each study. Additionally, there were studies that categorized cirrhosis based on the Child-Pugh score into A/B/C and performed associated detailed analyses. ${ }^{26-28,30,31,34,36}$

\section{Methodological quality and risk of bias in the included studies}

After using QUADAS-II, the tool for evaluating diagnostic accuracy, to evaluate the final 12 chosen studies, none of the studies were found to have a high risk of bias (Fig. 2). Although some of the studies selectively sampled the subjects, the method for selecting patients was judged to be of little concern, due to the fact that they were studies comparing the diagnostic accuracy of a healthy control group and a patient group. There were many studies in which the time difference between the performance of liver biopsy and CEUS was not reported or was unclear, ${ }^{13,26-29,31-33}$ where it was unclear whether reading of the results from the reference standard and CEUS were performed independently, ${ }^{13,26-28,31-36}$ or where the reports regarding the failure rate of test subjects were unclear. ${ }^{13,26-29,31-33}$ However, it must be taken into consideration that these related studies were published in journals that focused in radiology and did not place importance on those categories. Therefore, the selected studies were evaluated as being without risk of bias or problems with respect to the application and adherence to most of the requirements for quality evaluation (Fig. 2).

\section{Accuracy of HVAT assessed by CEUS}

The diagnostic accuracy of CEUS was compared to that of biopsy, a reference standard, to see how well it could distinguish subjects with cirrhosis, fibrosis, and nonfibrosis. The diagnostic accuracy results were described through total sensitivity, specificity, LR(+), LR(-), DOR, and SROC statistics.

In individual studies, CEUS was reported to have a 0.56 to
1.00 distribution in sensitivity and a 0.43 to 0.93 specificity in prediction of cirrhosis. Depending on the contrast agent used, the studies showed varying results. For example, studies that used first-generation contrast agents like Levovist (Schering $\mathrm{AG})^{13,27,28,32}$ had results (sensitivity, 1.0; specificity, 0.80 to 0.84 ) that did not vary much from studies that used a second-generation contrast agent like SonoVue or Sonazoid or Definity (sensitivity, 0.56 to 1.00 ; specificity, 0.43 to 0.93 )..$^{26,29-31,34-36}$ However, in cases where second-generation contrast agents were used, varying result values were found. Depending on the contrast agent used (SonoVue, Sonazoid, and Definity), the following results were reported: studies that used Sonazoid ${ }^{30}$ or Definity ${ }^{36}$ had resulting diagnosis accuracies (sensitivity, 0.56 to 0.57 ; specificity, 0.43 to 0.57 ) lower than those reported in studies that used SonoVue (sensitivity, 0.71 to 1.00 ; specificity, 0.57 to 0.93). ${ }^{26,29,35}$ Furthermore, studies ${ }^{13,26,28,30,36}$ that used US/Doppler to calculate the HVAT for the purpose of diagnosing cirrhosis and studies ${ }^{29,35}$ that used visual inspection as a means of assessment were compared. Upon comparing the results, the studies utilizing visual inspection reported lower diagnostic accuracy (sensitivity, 0.71 to 0.79 ; specificity, 0.57 to 0.79 ) than the resulting values of the data obtained using US/Doppler (sensitivity, 0.56 to 1.0 ; specificity, 0.43 to 0.93 ). A study ${ }^{26}$ that used SonoVue, the most widely used second-generation contrast agent at the time, as the contrast agent and US/Doppler to calculate the HVAT in order to evaluate the diagnostic accuracy of cirrhosis was reported to have produced the high results of sensitivity 1.0 and specificity 0.93 (Table 2).

Upon MA, in predicting cirrhosis based on seven studies ( $\mathrm{n}=469),{ }^{13,26,28-30,35,36}$ CEUS HVAT had an integrated sensitivity of 0.83 (95\% CI, 0.77 to 0.89) (Fig. 3A) and an integrated specificity of 0.75 (95\% CI, 0.69 to 0.79 ) (Fig. 3B). Among the studies, the heterogeneity value $\mathrm{I}^{2}$ for each was high at $84.3 \%\left(\mathrm{X}^{2}=38.34\right.$, $\mathrm{p}<0.001)$ and $89.6 \%\left(\mathrm{X}^{2}=57.48, \mathrm{p}<0.001\right)$. The integrated $\mathrm{LR}(+)$ was 3.45 (95\% CI, 1.60 to 7.43 ), while the integrated LR(-) was

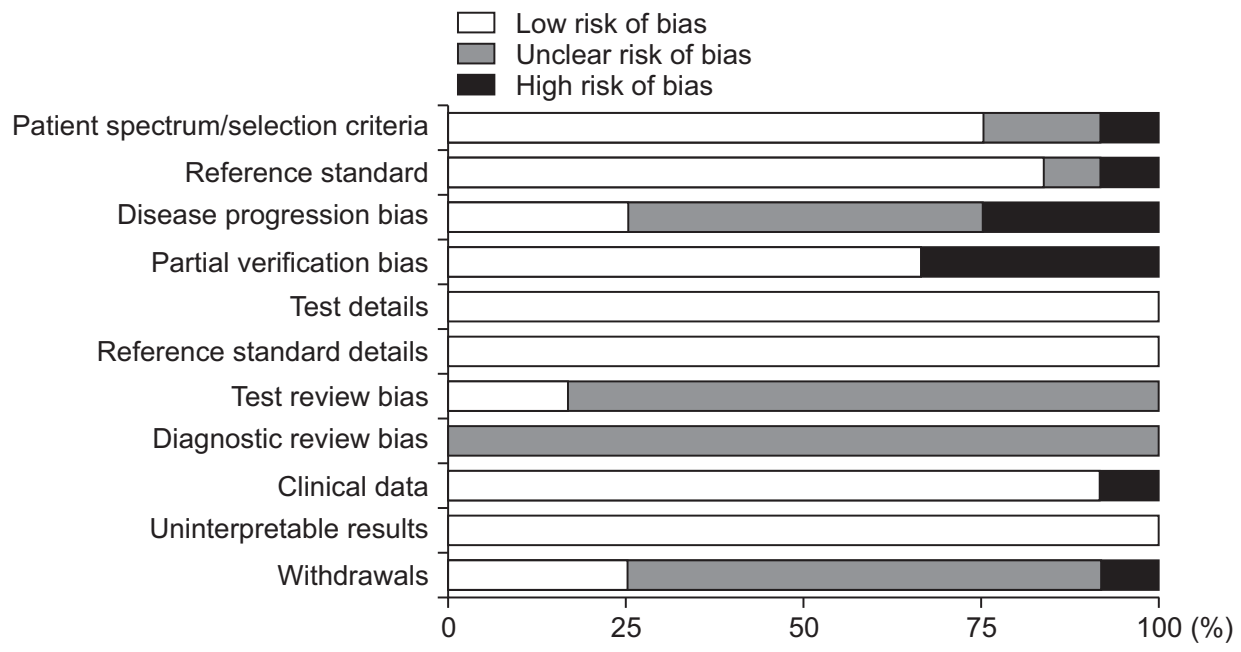

Fig. 2. The risk of bias and concerns regarding the applicability of the studies included in the analysis (quality assessment of diagnostic accuracy studies-II [QUADAS-II]). 
Table 2. The Accuracy of HVAT Assessed by CEUS

\begin{tabular}{|c|c|c|c|c|c|c|c|c|c|c|c|c|c|}
\hline \multirow{2}{*}{ Author (year) } & \multicolumn{4}{|c|}{$2 \times 2$ Table } & \multirow{2}{*}{$\begin{array}{l}\text { Cutoff } \\
\text { value }\end{array}$} & \multirow{2}{*}{$\begin{array}{c}\text { Se, } \\
\%\end{array}$} & \multirow{2}{*}{$\begin{array}{l}\mathrm{Sp}, \\
\%\end{array}$} & \multirow{2}{*}{$\begin{array}{c}\text { PPV, } \\
\% 0\end{array}$} & \multirow{2}{*}{$\begin{array}{c}\text { NPV, } \\
\%\end{array}$} & \multirow{2}{*}{$\begin{array}{l}\text { AUROC } \\
(95 \% \text { CI) }\end{array}$} & \multirow{2}{*}{$\begin{array}{c}\text { Accuracy, } \\
\%\end{array}$} & \multicolumn{2}{|c|}{ ICC } \\
\hline & $\mathrm{TP}$ & $\mathrm{FP}$ & FN & $\mathrm{TN}$ & & & & & & & & Intra & Inter \\
\hline Abbattista et al. $(2008)^{26}$ & 38 & 3 & 0 & 42 & $\leq 17$ & 100 & 93.3 & 92.6 & 100 & - & 96.3 & 1.0 & 0.93 \\
\hline Blomley et al. (2003) & 20 & 3 & 0 & 16 & $<24$ & 100 & 84.2 & 86.9 & 100 & - & 92.3 & - & - \\
\hline Cobbold et al. $(2012)^{29}$ & 12 & 25 & 5 & 33 & $<23.75$ & 71 & 57 & 32 & 87 & $0.71(0.55-0.86)$ & 60.0 & 0.99 & 0.94 \\
\hline Lim et al. $(2005)^{13}$ & 10 & 4 & 0 & 51 & $\leq 21$ & 100 & 80 & 74 & 95 & - & 85.7 & - & - \\
\hline Staub et al. $(2009)^{35}$ & 41 & 11 & 11 & 60 & $<13$ & 78.6 & 79.0 & 78.3 & 83.3 & $0.85(0.77-0.93)$ & 78.8 & - & 0.90 \\
\hline Tang et al. $(2011)^{36}$ & 4 & 20 & 4 & 15 & $<24$ & 57 & 43 & 17 & 83 & $0.56 \pm 0.12$ & 44.1 & 0.62 & 0.86 \\
\hline
\end{tabular}

HVAT, hepatic vein arrival time; CEUS, contrast-enhanced ultrasonography; TP, true positive; FP, false positive; FN, false negative; TN, true negative; Se, sensitivity; Sp, specificity; PPV, positive predictive value; NPV, negative predictive value; AUROC, area under the curve for the receiveroperating characteristic; CI, confidence interval; ICC, intra-class correlation coefficient.

A

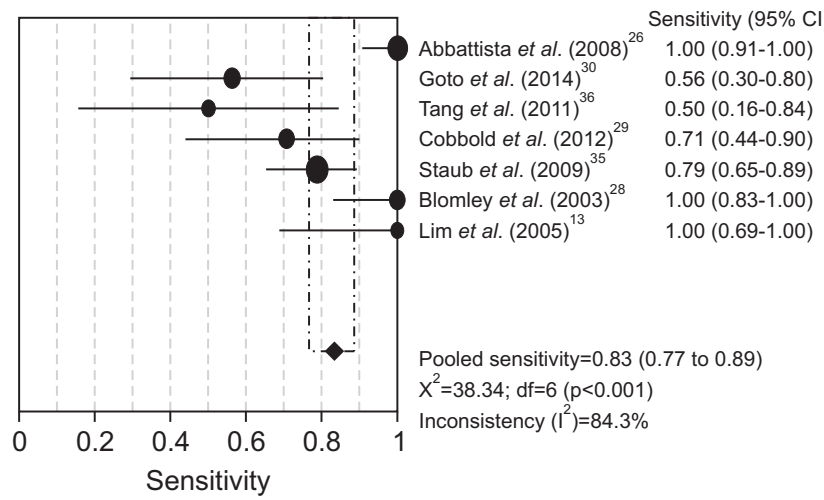

B

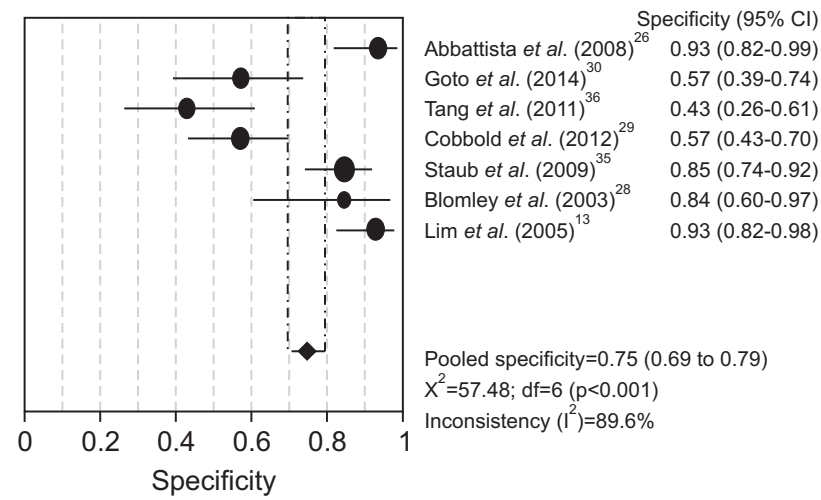

Fig. 3. Forest plot of sensitivity (A) and specificity (B) of hepatic vein arrival time assessed by contrast-enhanced ultrasonography for the detection of cirrhosis in the selected studies.

0.28 (95\% CI, 0.10 to 0.74). The summary DOR (random effects model) value was 15.23 (95\% CI, 3.07 to 75.47 ), $\mathrm{I}^{2}$ was $85.7 \%$ $\left(\mathrm{X}^{2}=41.96, \mathrm{p}<0.001\right)$, the SROC AUC was 0.74 (standard error $[\mathrm{SE}]=0.14)$, and index $\mathrm{Q}$ was $0.69(\mathrm{SE}=0.11)$.

The studies that used second-generation contrast agents and US/Doppler to calculate HVAT in order to predict cirrhosis had an integrated sensitivity of 0.82 (95\% CI, 0.71 to 0.91$)$ and an integrated specificity of 0.67 (95\% CI, 0.58 to 0.75$){ }^{26,30,36}$ The integrated LR(+) of these studies was 2.37 (95\% CI, 0.48 to 11.71), and the integrated LR(-) was 0.35 (95\% CI, 0.04 to 2.83). The summary DOR (random effects model) was 7.99 (95\% CI, 0.28 to 229.13), $I^{2}$ was $91.1 \%\left(X^{2}=22.37, p<0.001\right)$, the SROC AUC was $0.47(\mathrm{SE}=0.19)$, and index $\mathrm{Q}$ was 0.48 ( $\mathrm{SE}=0.15)$.

Furthermore, the studies that used second-generation contrast agents in order to predict cirrhosis had an integrated sensitivity of 0.79 (95\% CI, 0.71 to 0.86 ) and an integrated specificity of 0.70 (95\% CI, 0.64 to 0.75 ). ${ }^{26,29,30,35,36}$ The integrated LR(+) was 2.51 (95\% CI, 1.06 to 5.95), and the integrated LR(-) was 0.43 (95\% $\mathrm{CI}, 0.17$ to 1.08 ). The summary diagnostic odds ratio (random effects model) was 6.90 (95\% CI, 1.28 to 37.16 ), $\mathrm{I}^{2}$ was $87.1 \%$ $\left(\mathrm{X}^{2}=31.01, \mathrm{p}<0.001\right)$, the SROC AUC was 0.69 (SE=0.17), and index Q was 0.65 (SE=0.14).

Studies that used values obtained using Doppler to calculate the HVAT $^{13,26,28,30,36}$ had an integrated sensitivity of 0.88 (95\% CI, 0.80 to 0.94$)$ and an integrated specificity of 0.76 (95\% CI, 0.70 to 0.82$)$. The integrated $\mathrm{LR}(+)$ was 3.83 (95\% CI, 1.20 to 12.22 ), and the integrated LR(-) was 0.15 (95\% CI, 0.02 to 1.12). The summary DOR (random effects model) was 27.40 (95\% CI, 1.68 to 447.73$), \mathrm{I}^{2}$ was $88.9\left(\mathrm{X}^{2}=36.15, \mathrm{p}<0.001\right)$, the SROC AUC was 0.62 ( $\mathrm{SE}=0.20$ ), and index $\mathrm{Q}$ was 0.59 ( $\mathrm{SE}=0.16$ ) (Fig. 3).

\section{Correlation between HVAT assessed by CEUS and severity of liver fibrosis}

After studying a total of 12 studies on the relationship between stage of fibrosis and HVAT measured by CEUS, statistically significant decrease $(\mathrm{p}<0.05)$ was found between the HVAT of the normal or nonfibrosis groups (mean \pm standard deviation [SD], 34.63 \pm 10.27 ) and the HVATs of the fibrosis (SD, $25.01 \pm 5.46)$ and cirrhosis groups (SD, 17.62 \pm 3.57 ) showed (Table 3). 
Table 3. Correlation between HVAT Assessed by CEUS and Severity of Liver Fibrosis

\begin{tabular}{|c|c|c|c|}
\hline \multirow{2}{*}{ Author (year) } & \multicolumn{3}{|c|}{ HVAT, sec } \\
\hline & Normal or nonfibrosis & Fibrosis (mild to severe) & Overt cirrhosis \\
\hline Abbattista et al. $(2008)^{26}$ & $24.9 \pm 4.4$ & $21.7 \pm 3.5$ & $14.0 \pm 2.5$ \\
\hline Albrecht et al. (1999) ${ }^{27}$ & $49.8 \pm 22.6$ & $35.8 \pm 9.9$ & $18.3 \pm 3.0$ \\
\hline Blomley et al. $(2003)^{28}$ & $44.0 \pm 25.0$ & $26.0 \pm 8.0$ & $17.7 \pm 5.4$ \\
\hline Cobbold et al. $(2012)^{29}$ & - & $24.4(21.5-27.8)$ & $20.5(18.5-24.5)$ \\
\hline Goto et al. $(2014)^{30}$ & - & $27.4 \pm 1.7$ & $27.3 \pm 1.7$ \\
\hline Li et al. $(2010)^{31}$ & - & $25.1 \pm 4.1$ & $22.6 \pm 3.9$ \\
\hline Lim et al. $(2011)^{32}$ & $33.8 \pm 3.8$ & $29.7 \pm 2.2$ & $15.8 \pm 0.9$ \\
\hline Lim et al. $(2006)^{33}$ & $29.4 \pm 6.9$ & $25.2 \pm 7.0$ & $16.4 \pm 4.9$ \\
\hline Lim et al. $(2005)^{13}$ & $38.1 \pm 2.8$ & $32.4 \pm 2.4$ & $15.8 \pm 0.8$ \\
\hline Ridolfi et al. $(2007)^{34}$ & $24.8 \pm 4.4$ & $22.1 \pm 3.4$ & $14.3 \pm 2.1$ \\
\hline Staub et al. $(2009)^{35}$ & - & $15.3 \pm 5.4$ & $8.8 \pm 4.6$ \\
\hline Tang et al. $(2011)^{36}$ & - & $23.6 \pm 8.9$ & $20.9 \pm 5.5$ \\
\hline
\end{tabular}

HVAT, hepatic vein arrival time; CEUS, contrast-enhanced ultrasonography.

\section{DISCUSSION}

This is the first SR and MA of the utility of measuring HVAT by CEUS in cirrhosis. This study demonstrated that, because there is ample evidence disclosing a correlation between LB and HVAT, CEUS may have the potential to reduce the number of biopsy procedures performed to examine the severity of fibrosis. $^{13,26-36}$

Although biopsy is the most accurate test for diagnosing cirrhosis, patients with chronic liver disease require long-term follow-up. Consequently, a repeatedly available non-invasive method would be preferable for the assessment of hepatic fibrosis. $^{6,8}$

Indeed, ultrasonography has the advantage of being noninvasive, and many attempts have been made to assess the degree of hepatic fibrosis using ultrasonography. In particular, a US parameter that could be a suitable substitute for an invasive method for assessing hepatic fibrosis, such as the LB, would be highly desirable. However, these indices are plagued by lack of reproducibility and accuracy due to intra- and inter-observer variability and even inter-equipment variability.,6,8

Recently, the development of new contrast agents has increased the diagnostic capability of US, suggesting that the analysis of HVAT using microbubble CEUS can be useful for assessing the severity of liver fibrosis. ${ }^{10-13}$ HVAT is the time (in seconds) after injection for the microbubble contrast agent to arrive at the hepatic vein. ${ }^{2,3}$ There is an inverse relationship between HVAT and liver histological grade, such that a decrease in HVAT is accompanied by an increase in the severity of liver fibrosis, due to the formation of intrahepatic arteriovenous or portovenous shunts in the cirrhotic liver. ${ }^{2}$ Accordingly, HVAT as assessed by CEUS is negatively correlated with severity of hepatic fibrosis due to the associated hemodynamic alterations. ${ }^{11-13}$
Therefore, we reviewed the parameter of onset time of contrast enhancement in the right hepatic vein, which did show a significant correlation with degree of hepatic fibrosis.

The diagnostic accuracy of CEUS was determined to be high through the present SR and MA, with an integrated sensitivity of 0.83 (95\% CI, 0.77 to 0.89), a specificity of 0.75 (95\% CI, 0.69 to 0.79 ), and a SROC AUC of 0.74 ( $\mathrm{SE}=0.14$ ). However, the heterogeneity of the studies was also high $\left(\mathrm{I}^{2}, 84.3 \%\right.$ to $\left.89.6 \%\right)$. During analysis of the data, the selected studies were found to have used different microbubble contrast agents, each having their own chemical properties, including Levovist, SonoVue, Sonazoid, and DefinityIn this SR and MA, studies using Sonazoid and Definity showed lower diagnostic accuracies with relatively poor sensitivities and specificities compared to results obtained using Levovist and SonoVue. ${ }^{30,36}$ This finding can be explained by the finding that the HVAT is influenced by the microbubble extraction rate due to phagocytosis, particularly with respect to the 99\% rate of Sonazoid, suggesting that the higher extraction rate by Kupffer cells may accentuate this confounding influence in measuring HVAT. ${ }^{30}$ Unlike Sonazoid, SonoVue, a microbubble agent containing 2 to $10 \mu \mathrm{m}$ sulfur hexafluoride gas in a phopholipidic monolayer shell, undergoes less phagocytosis and remains mainly in the blood vessels, making it helpful when judging the special characteristics of the blood vessels. ${ }^{36}$ This suggests that, since HVAT is affected by phagocytosis of the contrast agent, these differences could possibly result in different signal intensities and transit times. Additional effort investigating differences in the interactions of Levovist, SonoVue, Sonazoid, and Definity with the reticuloendothelial system will be needed to explain these discrepant results.

In addition to HVAT, some studies have measured additional parameters, including the transit time between the hepatic artery and vein, and the slope gradient of each hepatic artery, portal 
vein, and hepatic vein. ${ }^{27,30,31}$ However, because the data utility of these various parameters using CEUS was too limited to conduct SR and MA, we chose the parameter of HVAT, which has been widely reported in the majority of studies related to this topic.

The present study has the following potential limitations. First, only 12 studies evaluated the usefulness of the CEUS on hepatic fibrosis, thus limiting the robustness of the conclusions that could be reached. Second, the characteristics of the included studies, including patient characteristics, etiologies of cirrhosis, and methodological differences, were not completely consistent. Third, we only included studies written in English, so language bias might have influenced the results.

In conclusion, the measurement of HVAT using CEUS reflected the severity of hepatic fibrosis. Additionally, this method seems to be simple and safe with its noninvasiveness. Hence, although this technique requires further investigation, we suggest that measuring HVAT by CEUS could be a reliable noninvasive supplementary tool for diagnosis of cirrhosis. Nevertheless, future large-scaled randomized controlled studies would be needed to draw out the firm conclusion.

\section{CONFLICTS OF INTEREST}

No potential conflict of interest relevant to this article was reported.

\section{ACKNOWLEDGEMENTS}

This research was supported by a grant of the Korea Health Technology R\&D Project through the Korea Health Industry Development Institute (KHIDI), funded by the Ministry of Health \& Welfare, Republic of Korea (HI15C2364), and also by the Yonsei University Future-Leading Research Initiative of 2014.

Authors' contributions: conception and design, G.K. and S.K.B.; acquisition of data, G.K.; analysis and interpretation of data, G.K. and S.K.B.; drafting the manuscript, G.K., S.K.B., and K.Y.S.; revising for intellectual content, G.K., S.K.B., and K.Y.S.; final approval and accountability for content, all authors.

\section{REFERENCES}

1. Eom YW, Shim KY, Baik SK. Mesenchymal stem cell therapy for liver fibrosis. Korean J Intern Med 2015;30:580-589.

2. Kim MY, Suk KT, Baik SK, et al. Hepatic vein arrival time as assessed by contrast-enhanced ultrasonography is useful for the assessment of portal hypertension in compensated cirrhosis. Hepatology 2012;56:1053-1062.

3. Baik SK. Haemodynamic evaluation by Doppler ultrasonography in patients with portal hypertension: a review. Liver Int 2010;30:1403-1413.

4. Hong WK, Shim KY, Baik SK, et al. Relationship between tetrahydrobiopterin and portal hypertension in patients with chronic liver disease. J Korean Med Sci 2014;29:392-399.

5. Kim JH, Kim JM, Cho YZ, et al. Effects of candesartan and propranolol combination therapy versus propranolol monotherapy in reducing portal hypertension. Clin Mol Hepatol 2014;20:376-383.

6. Kim MY, Jeong WK, Baik SK. Invasive and non-invasive diagnosis of cirrhosis and portal hypertension. World J Gastroenterol 2014;20:4300-4315.

7. Jang YO, Jun BG, Baik SK, Kim MY, Kwon SO. Inhibition of hepatic stellate cells by bone marrow-derived mesenchymal stem cells in hepatic fibrosis. Clin Mol Hepatol 2015;21:141-149.

8. Moon KM, Kim G, Baik SK, et al. Ultrasonographic scoring system score versus liver stiffness measurement in prediction of cirrhosis. Clin Mol Hepatol 2013;19:389-398.

9. Blomley MJ, Cooke JC, Unger EC, Monaghan MJ, Cosgrove DO. Microbubble contrast agents: a new era in ultrasound. BMJ 2001;322:1222-1225.

10. Jang JY, Kim MY, Jeong SW, et al. Current consensus and guidelines of contrast enhanced ultrasound for the characterization of focal liver lesions. Clin Mol Hepatol 2013;19:1-16.

11. Sugimoto H, Kaneko T, Hirota M, Tezel E, Nakao A. Earlier hepatic vein transit-time measured by contrast ultrasonography reflects intrahepatic hemodynamic changes accompanying cirrhosis. J Hepatol 2002;37:578-583.

12. Nicolau C, Ripollés T. Contrast-enhanced ultrasound in abdominal imaging. Abdom Imaging 2012;37:1-19.

13. Lim AK, Taylor-Robinson SD, Patel N, et al. Hepatic vein transit times using a microbubble agent can predict disease severity noninvasively in patients with hepatitis C. Gut 2005;54:128-133.

14. Kim G, Lee SS, Baik SK, et al. The need for histological subclassification of cirrhosis: a systematic review and meta-analysis. Liver Int 2016;36:847-855.

15. Kim G, Cho YZ, Baik SK. Assessment for risk of bias in systematic reviews and meta-analyses in the field of hepatology. Gut Liver 2015;9:701-706.

16. Kim G, Eom YW, Baik SK, et al. Therapeutic effects of mesenchymal stem cells for patients with chronic liver diseases: systematic review and meta-analysis. J Korean Med Sci 2015;30:1405-1415.

17. Kim G, Cho YZ, Baik SK, Kim MY, Hong WK, Kwon SO. The accuracy of ultrasonography for the evaluation of portal hypertension in patients with cirrhosis: a systematic review. Korean J Radiol 2015;16:314-324.

18. Kim G, Baik SK. Overview and recent trends of systematic reviews and meta-analyses in hepatology. Clin Mol Hepatol 2014;20:137150.

19. Higgins JP, Green S. Cochrane handbook for systematic reviews of interventions version 5.1.0 [Internet]. Copenhagen: The Cochrane Collaboration; [cited 2015 Aug 8]. Available from: http://handbook.cochrane.org/.

20. Moher D, Liberati A, Tetzlaff J, Altman DG; PRISMA Group. Preferred reporting items for systematic reviews and meta-analyses: the PRISMA statement. Ann Intern Med 2009;151:264-269.

21. Whiting PF, Rutjes AW, Westwood ME, et al. QUADAS-2: a re- 
vised tool for the quality assessment of diagnostic accuracy studies. Ann Intern Med 2011;155:529-536.

22. Deeks JJ, Macaskill P, Irwig L. The performance of tests of publication bias and other sample size effects in systematic reviews of diagnostic test accuracy was assessed. J Clin Epidemiol 2005;58:882-893.

23. Moses LE, Shapiro D, Littenberg B. Combining independent studies of a diagnostic test into a summary ROC curve: dataanalytic approaches and some additional considerations. Stat Med 1993;12:1293-1316.

24. Greiner M, Pfeiffer D, Smith RD. Principles and practical application of the receiver-operating characteristic analysis for diagnostic tests. Prev Vet Med 2000;45:23-41.

25. Walter SD. Properties of the summary receiver operating characteristic (SROC) curve for diagnostic test data. Stat Med 2002;21:1237-1256.

26. Abbattista T, Ridolfi F, Ciabattoni E, et al. Diagnosis of liver cirrhosis by transit-time analysis at contrast-enhanced ultrasonography. Radiol Med 2008;113:860-874.

27. Albrecht T, Blomley MJ, Cosgrove DO, et al. Non-invasive diagnosis of hepatic cirrhosis by transit-time analysis of an ultrasound contrast agent. Lancet 1999;353:1579-1583.

28. Blomley MJ, Lim AK, Harvey CJ, et al. Liver microbubble transit time compared with histology and Child-Pugh score in diffuse liver disease: a cross sectional study. Gut 2003;52:1188-1193.

29. Cobbold JF, Patel D, Fitzpatrick JA, et al. Accuracy and reliability of microbubble ultrasound measurements for the non-invasive assessment of hepatic fibrosis in chronic hepatitis C. Hepatol Res 2012;42:515-522.

30. Goto Y, Okuda K, Akasu G, Kinoshita H, Tanaka H. Noninvasive diagnosis of compensated cirrhosis using an analysis of the timeintensity curve portal vein slope gradient on contrast-enhanced ultrasonography. Surg Today 2014;44:1496-1505.

31. Li N, Ding H, Fan P, et al. Intrahepatic transit time predicts liver fibrosis in patients with chronic hepatitis B: quantitative assessment with contrast-enhanced ultrasonography. Ultrasound Med Biol 2010;36:1066-1075.

32. Lim AK, Patel N, Eckersley RJ, et al. A comparison of 31P magnetic resonance spectroscopy and microbubble-enhanced ultrasound for characterizing hepatitis c-related liver disease. J Viral Hepat 2011;18:e530-e534.

33. Lim AK, Patel N, Eckersley RJ, et al. Hepatic vein transit time of SonoVue: a comparative study with Levovist. Radiology 2006;240: 130-135.

34. Ridolfi F, Abbattista T, Marini F, et al. Contrast-enhanced ultrasound to evaluate the severity of chronic hepatitis C. Dig Liver Dis 2007;39:929-935.

35. Staub F, Tournoux-Facon C, Roumy J, et al. Liver fibrosis staging with contrast-enhanced ultrasonography: prospective multicenter study compared with METAVIR scoring. Eur Radiol 2009;19:19911997.

36. Tang A, Kim TK, Heathcote J, et al. Does hepatic vein transit time performed with contrast-enhanced ultrasound predict the severity of hepatic fibrosis? Ultrasound Med Biol 2011;37:1963-1969. 